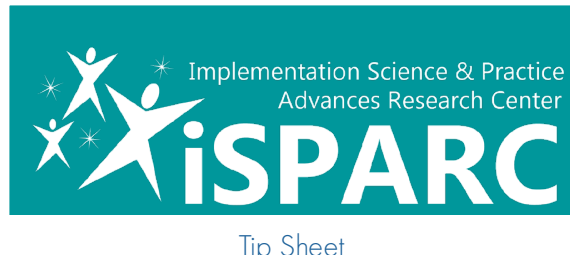

Tip Sheet

\title{
MAINTAINING YOUR EMOTIONAL WELLNESS DURING COVID-19
}

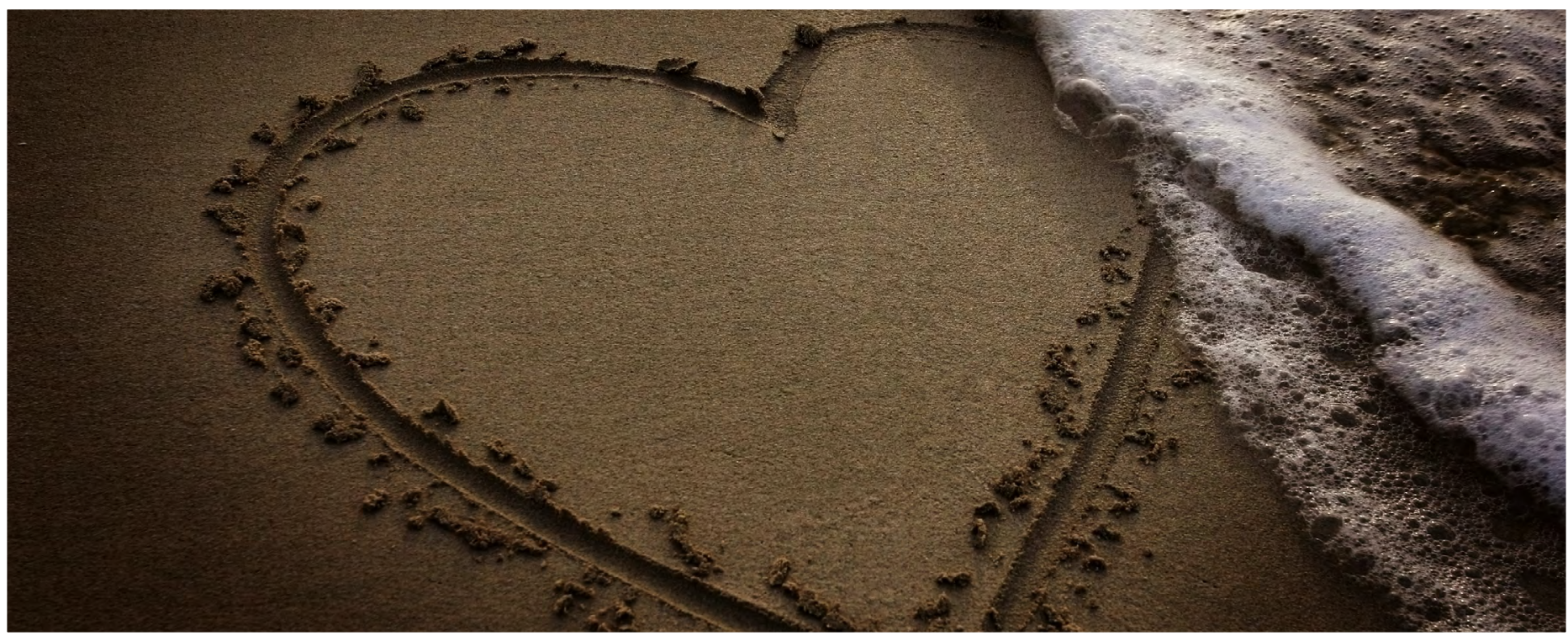

The world is a little scary and stressful right now because the COVID- 19 outbreak has impacted many people's lives in one way or another. Being anxious during a time like this is completely normal, but the fear and uncertainty can have a negative impact on your mental health. Now more than ever taking care of your mental health is a priority. We here at the Implementation Science and Practice Advances Research Center (iSPARC) have seen many great resources and tips come out to help people support their mental health during this crisis. We've pointed out some of the ones we think are the most helpful and listed them below. The sources of the tips are on the next page.

- Stick to your pre-COVID-19 routine as much as you can. Go to bed and wake up at reasonable times, shower, get dressed, and eat on a schedule. When eating, try to eat healthy; limiting junk food and sweets.

- Try to be active for at least 30 minutes each day. If you can, spend some time outside too while maintaining the appropriate distance from other people. You can still take walks, jog, bike ride, hike and more.

- Make sure to get news and information from credible sources like your local government authorities.

- Repeatedly hearing about the pandemic can be upsetting, so make sure to take breaks from news, social media, radio, etc.

- Take time to unwind and remind yourself that strong feelings will fade. Try taking in deep breaths. Try to do activities you usually enjoy. Maybe try something new or do some spring cleaning.

Continue any treatment you've been getting. Telepsych and telehealth are being made available, so work with your provider and health insurance to make sure your needs are being met. 
- Make sure you connect with friends or family members. Maintaining healthy relationships where you can share emotions and concerns will help you feel less alone and stressed.

- Seek help when you need it. If you have feelings or behaviors for several days in a row and are unable to carry out normal responsibilities because of them, get help. If you can't reach a provider, there are other options: (e.g.,

- SAMHSA Helpline 1-800-985-5990, text HOME to 741741 to reach a crisis counselor

- National Suicide Prevention line 1-800-273-8255

- Disaster Distress Helpline 1-800-985-5990 or text TalkWithUs to 66746).

\section{Online Resources}

- Brain \& Behavior Research Foundation. How to stay mentally healthy amidst the COVID-19 pandemic: https://www.bbrfoundation.org/blog/how-stay-mentally-healthy-amidst-covid-19-pandemic

- Care and Counseling. Tips and resources for taking care of your mental health during the COVID-19 pandemic: https:// careandcounseling.org/2020/03/20/tips-and-resources-for-taking-care-of-your-mental-healthduring-the-covid-19-pandemic/

- CDC. Coronavirus (COVID-19): https://www.cdc.gov/coronavirus/2019-ncov/index.html

- CDC. Coronavirus disease 2019 (COVID-19): Daily life \& coping: https://www.cdc.gov/ coronavirus/2019ncov/daily-life-coping/managing-stress-anxiety.html

- CDC. Taking care of your emotional health: https://emergency.cdc.gov/coping/selfcare.asp

- Mclean Hospital. Caring for your mental health despite the coronavirus: https:/ / www.mcleanhospital.org/ news/caring-your-mental-health-despite-coronavirus

- NIMH. Supporting mental health during the COVID-19 pandemic: https://www.nimh.nih.gov/news/ science-news/2020/supporting-mental-health-during-the-covid-19-pandemic.shtml

- SAMHSA. Taking care of your behavioral health: Tips for social distancing, quarantine, and isolation during an infectious disease outbreak: https://www.samhsa.gov/sites/default/files/tips-social-distancingquarantine-isolation-031620.pdf

- Simon Fraser University. Mental health wellness tips for quarantine: http://www.sfu.ca/olc/blog/my-ssp/ mental-health-wellness-tips-quarantine

- The Jed Foundation. COVID-19 and managing mental health: https://www.jedfoundation.org/covid-19and-managing-mental-health/

- The National Suicide Prevention Lifeline. Emotional wellbeing during the COVID-19 outbreak: https:// suicidepreventionlifeline.org/current-events/supporting-your-emotional-well-being-during-the-covid-19outbreak/

- World Health Organization. Mental health considerations during COVID-19 outbreak: https://d 19feca0eced-41 a5-87d8-6715b9022c27.usrfiles.com/ugd/eeeef8_430cbd1857804d5ea644a32c5dca8d71.pdf

This is a publication of the Implementation Science and Practice Advances Research Center (iSPARC) a Massachusetts Department of Mental Health Research Center of Excellence.

The contents of this tip sheet were supported in part with funding from the Massachusetts Department of Mental Health.

The contents of this tip sheet do not necessarily represent the policy of MA DMH, NIDILRR, ACL, or HHS and you should not assume endorsement by local or the Federal Government. 